International Journal of Pure and Applied Mathematics

Volume 106 No. 1 2016, 237-247

ISSN: 1311-8080 (printed version); ISSN: 1314-3395 (on-line version)

url: http://www.ijpam.eu

doi: 10.12732/ijpam.v106i1.18

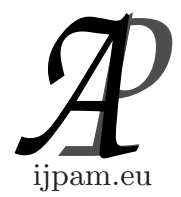

\title{
ON THE CONVERGENCE FOR THE SUM OF MONOTONE OPERATORS IN HILBERT SPACES
}

\author{
Chahn Yong Jung ${ }^{1}$, Shin Min Kang ${ }^{2}$ \\ ${ }^{1}$ Department of Business Administration \\ Gyeongsang National University \\ Jinju 52828, KOREA \\ ${ }^{2}$ Department of Mathematics and RINS \\ Gyeongsang National University \\ Jinju 52828, KOREA
}

\begin{abstract}
Let $C$ be a nonempty closed convex subset of a Hilbert space $H, A: C \rightarrow C$ be a nonexpansive mapping, $B: C \rightarrow H$ be a $\tau$-inverse strongly monotone mapping and $M$ be a maximal monotone operator on $H$ such that the domain of $M$ is included in $C$. In this paper, we prove the iterative sequence with errors converges weakly to a common element of $F(A)$ and $(B+M)^{-1} 0$ under the suitable conditions.
\end{abstract}

AMS Subject Classification: 47H05, 47H09, 46C05

Key Words: nonexpansive mapping, inverse strongly monotone mapping, maximal monotone operator, resolvent

\section{Introduction and Preliminaries}

Many theorems of the existence of fixed points on nonlinear operators have found and improved since 1922, when Banach proved a theorem, which is called Banach's fixed point theorem [1] (or Banach's contraction principle); see [6, 8, $9,18]$. Recently, some authors have studied on the convergence for the sum of monotone operators in Hilbert spaces, for instance, see [3, 7, 14].

Received: November 2, 2015

Published: February 4, 2016 (c) 2016 Academic Publications, Ltd.

url: www.acadpubl.eu 
Throughout this paper, we always assume that $H$ is a Hilbert space with inner product $\langle\cdot, \cdot\rangle$ and norm $\|\cdot\|$ and $C$ is a nonempty closed convex subset of $H$ to find a common element of a fixed point set of nonexpansive mappings and zeros of the sum of monotone operators under the suitable conditions.

Now, we are ready to introduce some important mappings which are used in the paper.

A mapping $A: C \rightarrow C$ is called nonexpansive if

$$
\|A x-A y\| \leq\|x-y\|, \quad \forall x, y \in C
$$

We denote by $F(A)$ the set of fixed points of $A$. If $C$ is nonempty bounded closed convex and $A: C \rightarrow C$ is nonexpansive, then $F(A)$ is nonempty. This explains why we assume the common element set used in main results is nonempty; for instance, see [16]. Moreover, if $C$ is closed and convex and $A: C \rightarrow C$ is nonexpansive, then it is well known that $F(A)$ is closed and convex and $I-A$ is demiclosed, that is, $\omega \in F(A)$ whenever $x_{n}-A x_{n} \rightarrow 0$ and $x_{n} \rightarrow \omega$. There are many iterative methods for approximation of fixed points of a nonexpansive mapping in a Hilbert space; see, for instance, [6, 15, 16, 17, 18].

A mapping $B$ of $C$ into $H$ is called inverse strongly monotone if there exists a positive real number $\tau$ such that

$$
\langle B x-B y, x-y\rangle \geq \tau\|B x-B y\|^{2}, \quad \forall x, y \in C .
$$

For such a case, $B$ is also said to be $\tau$-inverse strongly monotone.

For a nonempty closed convex subset $C$ of $H$, the nearest point projection of $H$ onto $C$ is denoted by $\operatorname{Proj}_{C}$, that is, $\left\|x-\operatorname{Proj}_{C} x\right\| \leq\|x-y\|, \forall x, y \in H$. Such $\operatorname{Proj}_{C}$ is called the metric projection of $H$ onto $C$. We know that the metric projection $\operatorname{Proj}_{C}$ is firmly nonexpansive, that is, $\left\|\operatorname{Proj}_{C} x-\operatorname{Proj}_{C} y\right\|^{2} \leq$ $\left\langle\operatorname{Proj}_{C} x-\operatorname{Proj}_{C} y, x-y\right\rangle, \forall x, y \in H$; see [15].

A multivalued operator $M: H \rightarrow 2^{H}$ with the domain $D(M)=\{x \in H$ : $M x \neq \emptyset\}$ and the range $R(M)=\{M x: x \in D(M)\}$ is said to be monotone if for each $x_{i} \in D(M)$ and $y_{i} \in M x_{i}, i=1,2$, we have $\left\langle x_{1}-x_{2}, y_{1}-y_{2}\right\rangle \geq 0$. A monotone operator $M$ is said to be maximal if its graph $G(M)=\{(x, y): y \in$ $M x\}$ is not properly contained in the graph of any other monotone operator. Let $I$ denote the identity operator on $H$ and $M: H \rightarrow 2^{H}$ be a maximal monotone operator. Then we can define, for each $\lambda>0$, a nonexpansive single valued mapping $J_{\lambda}^{M}: H \rightarrow H$ by $J_{\lambda}^{M}=(I+\lambda M)^{-1}$. It is called the resolvent of $M$. We know that $M^{-1} 0=F\left(J_{\lambda}^{M}\right)$ for all $\lambda>0$ and $J_{\lambda}^{M}$ is firmly nonexpansive; for instance, see $[2,3,4,5,12,13]$. 
In 1953, Mann [8] considered the following iterative scheme for finding a fixed point of a nonexpansive mapping $A$ of $H$ into itself,

$$
x_{n+1}=\alpha_{n} x_{n}+\left(1-\alpha_{n}\right) A x_{n}, \quad n=0,1,2, \cdots,
$$

where $x_{0}=x \in H$ and $\left\{\alpha_{n}\right\}$ is a sequence in $[0,1]$. He proved that the sequence $\left\{x_{n}\right\}$ generated by (1.1) converges weakly to some $z \in F(A)$.

Motivated by (1.1), Kamimura and Takahashi [7] introduced the following iterative scheme

$$
x_{n+1}=\alpha_{n} x_{n}+\left(1-\alpha_{n}\right) J_{\lambda_{n}}^{M} x_{n}, \quad n=0,1,2, \cdots,
$$

where $x_{0}=x \in H,\left\{\alpha_{n}\right\}$ is a sequence in $[0,1],\left\{\lambda_{n}\right\}$ is a sequence in $(0, \infty)$, $M: H \rightarrow 2^{H}$ is a maximal monotone and $J_{\lambda_{n}}^{M}=\left(I+\lambda_{n} M\right)^{-1}$. They showed that the sequence $\left\{x_{n}\right\}$ generated by (1.2) converges weakly to some $z \in M^{-1} 0$. Further, using this result, they investigated some algorithm in the case of $M=$ $\partial f$, where $f: H \rightarrow(-\infty, \infty]$ is a proper lower semicontinuous convex function. Define the subdifferential

$$
\partial f(x)=\{z \in H: f(x)+\langle y-x, z\rangle \leq f(y), \forall y \in H\}
$$

for all $x \in H$. It is well known that $\partial f$ is a maximal monotone operator of $H$ into itself; see [12].

Recently, Takahashi et al. [14] introduced the following iteration:

$$
x_{n+1}=\beta_{n} x_{n}+\left(1-\beta_{n}\right) A\left(\alpha_{n} x+\left(1-\alpha_{n}\right) J_{\lambda_{n}}^{M}\left(x_{n}-\lambda_{n} B x_{n}\right),\right.
$$

where $x_{1}=x \in C, B: C \rightarrow H$ is $\tau$-inverse strongly monotone, $M: D(M) \subset$ $C \rightarrow 2^{H}$ is maximal monotone, $J_{\lambda}^{M}=(I+\lambda M)^{-1}$ is the resolvent of $M$ for any $\lambda>0$ and $A: C \rightarrow C$ is a nonexpansive mapping. They proved that the sequence $\left\{x_{n}\right\}$ generated by (1.3) converges strongly to a point of $F(A) \cap(B+M)^{-1} 0$ under the suitable conditions.

In this paper, motivated by Takahashi et al. [14], we consider the convergence of an iterative sequence generated by a resolvent operator for a maximal monotone operator and nonlinear mappings in Hilbert spaces. With the help of the resolvent operator, we construct the iterative scheme to approximate the common element of a fixed point set for a nonexpansive mapping and a zeros of a mapping associated with a maximal monotone operator. Also, we obtain the weak convergence of the iteration with bounded errors.

Next, we collect several lemmas will be cited in the next section.

Lemma 1.1. ([2,3]) Properties of the resolvent $J_{\lambda}^{M}$ are listed as follows: 
(a) $\left\|J_{\lambda}^{M} x-J_{\lambda}^{M} y\right\|^{2} \leq\left\langle J_{\lambda}^{M} x-J_{\lambda}^{M} y, x-y\right\rangle, \forall x, y \in C$,

(b) $F\left(J_{\lambda}^{M}\right)=M^{-1} 0, \forall \lambda>0$,

(c) $J_{\lambda}^{M} x=J_{\mu}^{M}\left(\frac{\mu}{\lambda} x+\left(1-\frac{\mu}{\lambda}\right) J_{\lambda}^{M} x\right), \forall x \in H, \mu>0, \lambda>0$.

The property $(a)$ means the resolvent $J_{\lambda}^{M}$ is firmly nonexpensive and $(b)$ does the fixed point set of resolvent is right zeros of maximal monotone operator. The property $(c)$ is called resolvent identity.

Lemma 1.2. ([15]) Let $C$ be a nonempty closed convex subset of a real Hilbert space $H$ and $B: C \rightarrow H$ be $\tau$-inverse strongly monotone with a coefficient $\tau>0$. Then we have

$$
\|(I-\lambda B) x-(I-\lambda B) y\|^{2} \leq\|x-y\|^{2}+\lambda(\lambda-2 \tau)\|B x-B y\|^{2}, \quad \forall x, y \in C .
$$

If $0 \leq \lambda \leq 2 \tau$, then $I-\lambda B$ is nonexpansive.

Lemma 1.3. ([11]) Let $\left\{a_{n}\right\},\left\{b_{n}\right\}$ and $\left\{c_{n}\right\}$ be three nonnegative real number sequences satisfying the following condition:

$$
a_{n+1} \leq\left(1+b_{n}\right) a_{n}+c_{n}, \quad \forall n \geq n_{0} .
$$

where $n_{0}$ is some nonnegative integer, $\sum_{n=0}^{\infty} c_{n}<\infty$ and $\sum_{n=0}^{\infty} b_{n}<\infty$. Then $\lim _{n \rightarrow \infty} a_{n}$ exists.

Lemma 1.4. ([19]) Let $H$ be a Hilbert space. Then for each $x, y, z \in H$,

$$
\begin{aligned}
\|\alpha x+\beta y+\gamma z\|^{2} \leq & \alpha\|x\|^{2}+\beta\|y\|^{2}+\gamma\|z\|^{2}-\alpha \beta\|x-y\|^{2} \\
& -\beta \gamma\|y-z\|^{2}-\alpha \gamma\|x-z\|^{2}
\end{aligned}
$$

where $\alpha+\beta+\gamma=1$.

Throughout this paper, we already assumed $C$ is a nonempty closed convex subset of a real Hilbert space $H$. We know that $H$ satisfies Opial's property [10], that is, for any sequence $\left\{x_{n}\right\} \subset H$ with $x_{n} \rightarrow x$, the inequality $\liminf _{n \rightarrow \infty}\left\|x_{n}-x\right\|<\liminf _{n \rightarrow \infty}\left\|x_{n}-y\right\|$ holds for every $y \in H$ with $y \neq x$.

\section{Main Results}

Theorem 2.1. Let $C$ be a nonempty closed convex subset of a real Hilbert space $H, A: C \rightarrow C$ be a nonexpansive mapping, $B: C \rightarrow H$ be a $\tau$-inverse strongly monotone mapping and $M$ be a maximal monotone operator on $H$ such 
that the domain of $M$ is included in $C$. Assume that $\mathcal{F}=F(A) \cap(B+M)^{-1} 0 \neq$ $\emptyset$. Let $J_{\lambda_{n}}^{M}=\left(I+\lambda_{n} M\right)^{-1}$ be the resolvent of $M$ for any $\lambda_{n}>0$ and $\left\{e_{n}\right\}$ be a bounded error sequence in $C$. Let $\left\{x_{n}\right\}$ be a sequence in $C$ generated by $x_{1}=x \in C$ and

$$
\left\{\begin{array}{l}
y_{n}=\delta_{n} x_{n}+\left(1-\delta_{n}\right) A x_{n} \\
x_{n+1}=\alpha_{n} y_{n}+\beta_{n}\left(J_{\lambda_{n}}^{M}\left(x_{n}-\lambda_{n} B x_{n}\right)\right)+\gamma_{n} e_{n}
\end{array}\right.
$$

for all $n \in \mathbb{N}$, where $\left\{\alpha_{n}\right\},\left\{\beta_{n}\right\},\left\{\gamma_{n}\right\} \in(0,1)$ and $\alpha_{n}+\beta_{n}+\gamma_{n}=1$.

Assume that $\left\{\alpha_{n}\right\},\left\{\beta_{n}\right\},\left\{\gamma_{n}\right\},\left\{\delta_{n}\right\}$ and $\left\{\lambda_{n}\right\}$ satisfy the following:

(a) $0<a \leq \alpha_{n}<1$

(b) $0<b \leq \beta_{n} \leq c<1$,

(c) $\sum_{n=1}^{\infty} \gamma_{n}<\infty$

(d) $0<\delta_{n} \leq d<1$,

(e) $0<e \leq \lambda_{n} \leq f<2 \tau$,

where $a, b, c, d, e$ and $f$ are positive real numbers.

Then $\left\{x_{n}\right\}$ generated by $(2.1)$ converges weakly to a point of $\mathcal{F}$.

Proof. From Lemma 1.2, we know $I-\lambda_{n} B$ is nonexpansive. Actually, for any $x, y \in C$,

$$
\begin{aligned}
& \left\|\left(I-\lambda_{n} B\right) x-\left(I-\lambda_{n} B\right) y\right\|^{2} \\
& =\left\|(x-y)-\lambda_{n}(B x-B y)\right\|^{2} \\
& =\|x-y\|^{2}-2 \lambda_{n}\langle x-y, B x-B y\rangle+\lambda_{n}{ }^{2}\|B x-B y\|^{2} \\
& \leq\|x-y\|^{2}-2 \tau \lambda_{n}\|B x-B y\|^{2}+\lambda_{n}{ }^{2}\|B x-B y\|^{2} \\
& =\|x-y\|^{2}+\lambda_{n}\left(\lambda_{n}-2 \tau\right)\|B x-B y\|^{2} .
\end{aligned}
$$

From $(e)$, we get $\left\|\left(I-\lambda_{n} B\right) x-\left(I-\lambda_{n} B\right) y\right\|^{2} \leq\|x-y\|^{2}$, that is, $I-\lambda_{n} B$ is nonexpansive. Take $p \in \mathcal{F}$. Then $p \in F(A)$ and $p \in(B+M)^{-1} 0$.

From Lemma 1.1, we get

$$
p=A p=J_{\lambda_{n}}^{M}\left(p-\lambda_{n} B p\right)
$$


Put $u_{n}=J_{\lambda_{n}}^{M}\left(x_{n}-\lambda_{n} B x_{n}\right)$. Since $J_{\lambda_{n}}^{M}$ and $I-\lambda_{n} B$ are nonexpansive, we have

$$
\begin{aligned}
\left\|u_{n}-p\right\| & =\left\|J_{\lambda_{n}}^{M}\left(x_{n}-\lambda_{n} B x_{n}\right)-J_{\lambda_{n}}\left(p-\lambda_{n} B p\right)\right\| \\
& \leq\left\|\left(x_{n}-\lambda_{n} B x_{n}\right)-\left(p-\lambda_{n} B p\right)\right\| \\
& =\left\|\left(I-\lambda_{n} B\right) x_{n}-\left(I-\lambda_{n} B\right) p\right\| \\
& \leq\left\|x_{n}-p\right\| .
\end{aligned}
$$

Let $A_{n}=\delta_{n} I+\left(1-\delta_{n}\right) A$. Then $y_{n}=A_{n} x_{n}$ and $F(A)=F\left(A_{n}\right)$. It is easy to show that $A_{n}$ is nonexpansive. Hence we have

$$
\begin{aligned}
\left\|y_{n}-p\right\| & =\left\|A_{n} x_{n}-p\right\|=\left\|A_{n} x_{n}-A p\right\| \\
& =\left\|A_{n} x_{n}-A_{n} p\right\| \leq\left\|x_{n}-p\right\| .
\end{aligned}
$$

From (2.2) and (2.3), we have

$$
\begin{aligned}
\left\|x_{n+1}-p\right\| & =\left\|\alpha_{n}\left(y_{n}-p\right)+\beta_{n}\left(u_{n}-p\right)+\gamma_{n}\left(e_{n}-p\right)\right\| \\
& \left.\leq \alpha_{n}\left\|y_{n}-p\right\|+\beta_{n} \| u_{n}-p\right)\left\|+\gamma_{n}\right\| e_{n}-p \| \\
& \leq\left\|x_{n}-p\right\|+\gamma_{n}\left\|e_{n}-p\right\| .
\end{aligned}
$$

From Lemma 1.3, we see that $\lim _{n \rightarrow \infty}\left\|x_{n}-p\right\|$ exists and so $\left\{x_{n}\right\}$ is bounded. Hence $\left\{y_{n}\right\},\left\{u_{n}\right\}$ and $\left\{B x_{n}\right\}$ are so.

Using Lemma 1.4, we get

$$
\begin{aligned}
& \left\|x_{n+1}-p\right\|^{2} \\
& =\left\|\alpha_{n}\left(y_{n}-p\right)+\beta_{n}\left(u_{n}-p\right)+\gamma_{n}\left(e_{n}-p\right)\right\|^{2} \\
& \leq \alpha_{n}\left\|y_{n}-p\right\|^{2}+\beta_{n}\left\|u_{n}-p\right\|^{2}+\gamma_{n}\left\|e_{n}-p\right\|^{2} \\
& \quad-\alpha_{n} \beta_{n}\left\|y_{n}-u_{n}\right\|^{2}-\alpha_{n} \gamma_{n}\left\|y_{n}-e_{n}\right\|^{2}-\gamma_{n} \beta_{n}\left\|e_{n}-u_{n}\right\|^{2} \\
& \leq\left\|x_{n}-p\right\|^{2}-\alpha_{n} \beta_{n}\left\|y_{n}-u_{n}\right\|^{2}+\gamma_{n}\left\|e_{n}-p\right\|^{2},
\end{aligned}
$$

which yields

$$
\alpha_{n} \beta_{n}\left\|y_{n}-u_{n}\right\|^{2} \leq\left\|x_{n}-p\right\|^{2}-\left\|x_{n+1}-p\right\|^{2}+\gamma_{n}\left\|e_{n}-p\right\|^{2} .
$$

From the existence of $\lim _{n \rightarrow \infty}\left\|x_{n}-p\right\|$ and the conditions $(a)$ and $(b)$, we obtain

$$
\lim _{n \rightarrow \infty}\left\|y_{n}-u_{n}\right\|=0
$$


Notice

$$
\begin{aligned}
& \left\|u_{n}-p\right\|^{2} \\
& =\left\|J_{\lambda_{n}}^{M}\left(x_{n}-\lambda_{n} B x_{n}\right)-J_{\lambda_{n}}^{M}\left(p-\lambda_{n} B p\right)\right\|^{2} \\
& \leq\left\|\left(x_{n}-\lambda_{n} B x_{n}\right)-\left(p-\lambda_{n} B p\right)\right\|^{2} \\
& =\left\|x_{n}-p\right\|^{2}-2 \lambda_{n}\left\langle x_{n}-p, B x_{n}-B p\right\rangle+\lambda_{n}{ }^{2}\left\|B x_{n}-B p\right\|^{2} \\
& \leq\left\|x_{n}-p\right\|^{2}-2 \tau \lambda_{n}\left\|B x_{n}-B p\right\|^{2}+\lambda_{n}{ }^{2}\left\|B x_{n}-B p\right\|^{2} \\
& =\left\|x_{n}-p\right\|^{2}+\lambda_{n}\left(\lambda_{n}-2 \tau\right)\left\|B x_{n}-B p\right\|^{2} .
\end{aligned}
$$

By substituting (2.6), we have

$$
\begin{aligned}
& \left\|x_{n+1}-p\right\|^{2} \\
& \leq \alpha_{n}\left\|y_{n}-p\right\|^{2}+\beta_{n}\left\|u_{n}-p\right\|^{2}+\gamma_{n}\left\|e_{n}-p\right\|^{2} \\
& \leq \alpha_{n}\left\|x_{n}-p\right\|^{2}+\gamma_{n}\left\|e_{n}-p\right\|^{2} \\
& +\beta_{n}\left\{\left\|x_{n}-p\right\|^{2}+\lambda_{n}\left(\lambda_{n}-2 \tau\right)\left\|B x_{n}-B p\right\|^{2}\right\} \\
& \leq\left\|x_{n}-p\right\|^{2}+\gamma_{n}\left\|e_{n}-p\right\|^{2} \\
& +\beta_{n}\left\{\left\|x_{n}-p\right\|^{2}+\lambda_{n}\left(\lambda_{n}-2 \tau\right)\left\|B x_{n}-B p\right\|^{2}\right\},
\end{aligned}
$$

which derives the inequality

$$
\begin{gathered}
\beta_{n}\left\{\left\|x_{n}-p\right\|^{2}+\lambda_{n}\left(2 \tau-\lambda_{n}\right)\left\|B x_{n}-B p\right\|^{2}\right\} \\
\leq\left\|x_{n}-p\right\|^{2}-\left\|x_{n+1}-p\right\|^{2}+\gamma_{n}\left\|e_{n}-p\right\|^{2} .
\end{gathered}
$$

From the conditions $(b)$ and $(e)$, we obtain

$$
\lim _{n \rightarrow \infty}\left\|B x_{n}-B p\right\|=0 .
$$

We know the resolvent $J_{\lambda_{n}}^{M}$ of $M$ is firmly nonexpansive and $B$ is monotone. From this, we get the following

$$
\begin{aligned}
\left\|u_{n}-p\right\|^{2} \leq & \left\langle\left(x_{n}-\lambda_{n} B x_{n}\right)-\left(p-\lambda_{n} B p\right), u_{n}-p\right\rangle \\
= & \left\langle\left(x_{n}-p\right)-\lambda_{n}\left(B x_{n}-B p\right), u_{n}-p\right\rangle \\
= & \frac{1}{2}\left\{\left\|\left(x_{n}-p\right)-\lambda_{n}\left(B x_{n}-B p\right)\right\|^{2}+\left\|u_{n}-p\right\|^{2}\right. \\
& \left.-\left\|\left(x_{n}-p\right)-\lambda_{n}\left(B x_{n}-B p\right)-\left(u_{n}-p\right)\right\|^{2}\right\} \\
= & \frac{1}{2}\left\{\left\|x_{n}-p\right\|^{2}-2 \lambda_{n}\left\langle x_{n}-p, B x_{n}-B p\right\rangle+\left\|u_{n}-p\right\|^{2}\right. \\
& \left.-\left\|x_{n}-u_{n}\right\|^{2}+2 \lambda_{n}\left\langle x_{n}-u_{n}, B x_{n}-B p\right\rangle\right\} \\
\leq & \frac{1}{2}\left\{\left\|x_{n}-p\right\|^{2}+\left\|u_{n}-p\right\|^{2}-\left\|x_{n}-u_{n}\right\|^{2}\right. \\
& \left.+2 \lambda_{n}\left\|x_{n}-u_{n}\right\|\left\|B x_{n}-B p\right\|\right\}
\end{aligned}
$$


which yields

$$
\left\|u_{n}-p\right\|^{2} \leq\left\|x_{n}-p\right\|^{2}-\left\|x_{n}-u_{n}\right\|^{2}+2 \lambda_{n}\left\|x_{n}-u_{n}\right\|\left\|B x_{n}-B p\right\| .
$$

By substituting (2.8), we have

$$
\begin{aligned}
\left\|x_{n+1}-p\right\|^{2}= & \left\|\alpha_{n}\left(y_{n}-p\right)+\beta_{n}\left(u_{n}-p\right)+\gamma_{n}\left(e_{n}-p\right)\right\|^{2} \\
\leq & \left.\alpha_{n}\left\|y_{n}-p\right\|^{2}+\beta_{n} \| u_{n}-p\right)\left\|^{2}+\gamma_{n}\right\| e_{n}-p \|^{2} \\
\leq & \alpha_{n}\left\|x_{n}-p\right\|+\beta_{n}\left\{\left\|x_{n}-p\right\|^{2}-\left\|x_{n}-u_{n}\right\|^{2}\right. \\
& \left.+2 \lambda_{n}\left\|x_{n}-u_{n}\right\|\left\|B x_{n}-B p\right\|\right\}+\gamma_{n}\left\|e_{n}-p\right\| \\
\leq & \left\|x_{n}-p\right\|-\beta_{n}\left\|x_{n}-u_{n}\right\|^{2}+\gamma_{n}\left\|e_{n}-p\right\| \\
& +2 \beta_{n} \lambda_{n}\left\|x_{n}-u_{n}\right\|\left\|B x_{n}-B p\right\| .
\end{aligned}
$$

It follows that

$$
\begin{aligned}
\beta_{n}\left\|x_{n}-u_{n}\right\|^{2} \leq & \left\|x_{n}-p\right\|-\left\|x_{n+1}-p\right\|^{2}+\gamma_{n}\left\|e_{n}-p\right\| \\
& +2 \beta_{n} \lambda_{n}\left\|x_{n}-u_{n}\right\|\left\|B x_{n}-B p\right\| .
\end{aligned}
$$

From the conditions $(b),(c)$ and (2.7), we obtain

$$
\lim _{n \rightarrow \infty}\left\|x_{n}-u_{n}\right\|=0 .
$$

We know

$$
\left\|x_{n}-y_{n}\right\| \leq\left\|x_{n}-u_{n}\right\|+\left\|u_{n}-y_{n}\right\| .
$$

From (2.5) and (2.9), we get

$$
\lim _{n \rightarrow \infty}\left\|x_{n}-y_{n}\right\|=0
$$

Notice

$$
\begin{aligned}
\left\|A x_{n}-x_{n}\right\| & \leq\left\|A x_{n}-y_{n}\right\|+\left\|y_{n}-x_{n}\right\| \\
& =\delta_{n}\left\|A x_{n}-x_{n}\right\|+\left\|y_{n}-x_{n}\right\| .
\end{aligned}
$$

From this, we get

$$
\left(1-\delta_{n}\right)\left\|A x_{n}-x_{n}\right\| \leq\left\|y_{n}-x_{n}\right\| .
$$

From (2.10) and the condition $(d)$, we get

$$
\lim _{n \rightarrow \infty}\left\|A x_{n}-x_{n}\right\|=0 .
$$

Since $\left\{x_{n}\right\}$ is bounded, there exists a subsequence $\left\{x_{n_{i}}\right\}$ of $\left\{x_{n}\right\}$ such that $x_{n_{i}} \rightarrow \omega \in C$. 
First, we show $\omega \in F(A)$. Since $I-A$ demiclosed at 0 , we can get $\omega \in F(A)$. Next, we show $\omega \in(B+M)^{-1} 0$.

Notice

$$
\begin{aligned}
u_{n} & =J_{\lambda_{n}}\left(x_{n}-\lambda_{n} B x_{n}\right) \\
& \Leftrightarrow x_{n}-\lambda_{n} B x_{n} \in\left(I+\lambda_{n} M\right) u_{n} \\
& \Leftrightarrow \frac{x_{n}-u_{n}}{\lambda_{n}}-B x_{n} \in M u_{n} .
\end{aligned}
$$

Since $M$ is monotone, we get for any $(u, v) \in M$,

$$
\left\langle u_{n}-u, \frac{x_{n}-u_{n}}{\lambda_{n}}-B x_{n}-v\right\rangle \geq 0 .
$$

Replacing $n$ by $n_{i}$ and letting $i \rightarrow \infty$, we obtain

$$
\langle\omega-u,-(B \omega+v) \leq 0
$$

This means $-B \omega \in M \omega$, that is, $0 \in(B+M) \omega$. Hence we get $\omega \in(B+M)^{-1} 0$. Therefore, $\omega \in \mathcal{F}$.

Suppose that there exists another subsequence $\left\{x_{n_{j}}\right\}$ of $\left\{x_{n}\right\}$ such that $x_{n_{j}} \rightarrow \omega^{*}$. Then we can show $\omega^{*} \in \mathcal{F}$ in exactly the same way. Assume that $\omega \neq \omega^{*}$. Then we have

$$
\begin{aligned}
\lim _{n \rightarrow \infty}\left\|x_{n}-\omega\right\| & =\liminf _{i \rightarrow \infty}\left\|x_{n_{i}}-\omega\right\|<\liminf _{i \rightarrow \infty}\left\|x_{n_{i}}-\omega^{*}\right\| \\
& =\liminf _{j \rightarrow \infty}\left\|x_{n_{j}}-\omega^{*}\right\|<\liminf _{j \rightarrow \infty}\left\|x_{n_{j}}-\omega\right\| \\
& =\lim _{n \rightarrow \infty}\left\|x_{n}-\omega\right\| .
\end{aligned}
$$

This is a contraction. Therefore $\omega=\omega^{*}$ and $x_{n} \rightarrow \omega \in \mathcal{F}$. This completes the proof.

\section{Applications}

In this section, we draw some results using a proper lower semicontinuous convex function. Let $f: H \rightarrow(-\infty, \infty]$ be a proper lower semicontinuous convex function. Define the subdifferential $\partial f(x)$ of $f$ by

$$
\partial f(x)=\{z \in H: f(x)+\langle y-x, z\rangle \leq f(y), \forall y \in H\}
$$


for all $x \in H$. Then $\partial f$ is a maximal monotone operator of $H$ into itself. It is well-known by Rockafellar [17]. Let $C$ be a nonempty closed convex subset of $H$ and $i_{C}$ be the indicator function of $C$, that is,

$$
i_{C} x= \begin{cases}0, & x \in C, \\ \infty, & x \notin C .\end{cases}
$$

Furthermore, we define the normal cone $N_{C}(v)$ of $C$ at $v$ as follows:

$$
N_{C} v=\{z \in H:\langle z, y-v\rangle \leq 0, \forall y \in H\}
$$

for any $v \in C$. Then $i_{C}: H \rightarrow(-\infty, \infty]$ is a proper lower semicontinuous convex function on $H$ and $\partial i_{C}$ is a maximal monotone operator. Let $J_{\lambda_{n}}^{\partial i_{C}} x=$ $\left(I+\lambda_{n} \partial i_{C}\right)^{-1} x$ for any $\lambda_{n}>0$ and $x \in H$. From the facts $\partial i_{C} x=N_{C} x$ and $x \in C$, we get

$$
\begin{aligned}
v & =J_{\lambda_{n}}^{\partial i_{C}} x \Leftrightarrow\left(I+\partial i_{C}\right)^{-1} x \\
& =v \Leftrightarrow x \in v+\lambda_{n} N_{C} v \\
& \Leftrightarrow\langle x-v, y-v\rangle \leq 0, \forall y \in C \\
& \Leftrightarrow v=\operatorname{Proj}_{C} x,
\end{aligned}
$$

where $\operatorname{Proj}_{C}$ is the metric projection from $H$ into $C$. Similarly, we can get that $x \in\left(B+\partial i_{C}\right)^{-1} 0 \Leftrightarrow x \in V I(B, C)=\{z:\langle B z, y-z\rangle \geq 0, \forall y \in C\}$.

Put $M=\partial i_{C}$ in Theorem 2.1. Then we can see $J_{\lambda_{n}}^{M}=\operatorname{Proj}_{C}$ and conclude the following theorem.

Theorem 3.1. Let $C$ be a nonempty closed convex subset of a real Hilbert space $H, A: C \rightarrow C$ be a nonexpansive mapping and $B: C \rightarrow H$ be a $\tau$-inverse strongly monotone mapping such that $\mathcal{F}=F(A) \cap\left(B+\partial i_{C}\right)^{-1} 0 \neq \emptyset$. Let $\left\{e_{n}\right\}$ be a bounded error sequence in $C$. Let $\left\{x_{n}\right\}$ be a sequence in $C$ generated by $x_{1}=x \in C$ and

$$
x_{n+1}=\alpha_{n} A x_{n}+\beta_{n}\left(\operatorname{Proj}_{C}\left(x_{n}-\lambda_{n} B x_{n}\right)\right)+\gamma_{n} e_{n}
$$

for all $n \in \mathbb{N}$, where $\left\{\alpha_{n}\right\},\left\{\beta_{n}\right\},\left\{\gamma_{n}\right\} \in(0,1)$ and $\alpha_{n}+\beta_{n}+\gamma_{n}=1$.

Assume that $\left\{\beta_{n}\right\},\left\{\gamma_{n}\right\}$ and $\left\{\lambda_{n}\right\}$ satisfy the following:

(A) $0<a \leq \beta_{n} \leq b<1$,

(B) $0<c \leq \lambda_{n} \leq d<2 \alpha$,

(C) $\sum_{n=1}^{\infty} \gamma_{n}<\infty$

where $a, b, c$ and $d$ are positive real numbers.

Then the sequence $\left\{x_{n}\right\}$ generated by (3.1) converges weakly to a point of $\mathcal{F}$. 


\section{Acknowledgement}

This work (RPP-2014-020) was supported by the Fund of Research Promotion Program, Gyeongsang National University, 2014.

\section{References}

[1] S. Banach, Sur les opérations dans les ensembles abstraits et leur application aux équations intégrales, Fund. Math., 3 (1992), 133-181.

[2] V. Barbu, Nonlinear Semigroups and Differential Equations in Banach Spaces, Noordhoff, Leyden (1976).

[3] S.Y. Cho, Strong convergence of an iterative algorithm for sums of two monotone operators, J. Fixed Point Theory, 2013 (2013), Article ID 6, 8 pages.

[4] G. Crombez, Image recovery by convex combinations of projections, J. Math. Anal. Appl., 155 (1991), 413-419. doi: 10.1016/0022-247X(91)90010-W

[5] K. Eshita, W. Takahashi, Approximating zero points of accretive operators in general Banach spaces, JP J. Fixed Point Theory Appl., 2 (2007), 105-116.

[6] B. Halpern, Fixed points of nonexpanding maps, Bull. Amer. Math. Soc., 73 (1967), 957-961. doi: 10.1090/S0002-9904-1967-11864-0

[7] S. Kamimura, W. Takahashi, Approximating solutions of maximal monotone operators in Hilbert spaces, J. Approx. Theory, 106 (2000), 226-240. doi: 10.1006/jath.2000.3493

[8] W.R. Mann, Mean value methods in iteration, Proc. Amer. Math. Soc., 4 (1953), 506-510. doi: 10.1090/S0002-9939-1953-0054846-3

[9] A. Moudafi, Viscosity approximation methods for fixed-point problems, J. Math. Anal. Appl., 241 (2000), 46-55. doi: 10.1006/jmaa.1999.6615

[10] Z. Opial, Weak convergence of the sequence of successive approximations for nonexpansive mappings, Bull. Amer. Math. Soc., 73 (1967), 591-597. doi: 10.1090/S0002-99041967-11761-0

[11] M.O. Osilike, S.C. Aniagbosor, Weak and strong convergence theorems for fixed points of asymptotically nonexpansive mappings, Math. Comput. Modelling, 32 (2000), 1181-1191. doi: S0895-7177(00)00199-0

[12] R.T. Rockafellar, On the maximal monotonicity of subdifferential mappings, Pacific J. Math., 33 (1970), 209-216. doi: 10.2140/pjm.1970.33.209

[13] R.T. Rockafellar, Monotone operators and the proximal point algorithm, SIAM J. Control Optim., 14 (1976), 877-898. doi: 10.1137/0314056

[14] S. Takahashi, W. Takahashi, M. Toyoda, Strong convergence theorems for maximal monotone operators with nonlinear mappings in Hilbert spaces, J. Optim. Theory Appl., 147 (2010), 27-41. doi: 10.1007/s10957-010-9713-2

[15] W. Takahashi, Nonlinear Functional Analysis, Fixed Point Theory and its Applications, Yokohama Publishers, Yokohama (2000).

[16] W. Takahashi, Introduction to Nonlinear and Convex Analysis, Yokohama Publishers, Yokohama (2009). 
[17] W. Takahashi, M. Toyoda, Weak convergence theorems for nonexpansive mappings and monotone mappings, J. Optim. Theory Appl., 118 (2003), 417-428. doi: 10.1023/A:1025407607560

[18] R. Wittmann, Approximation of fixed points of nonexpansive mappings, Arch. Math., 58 (1992), 486-491. doi: 10.1007/BF01190119

[19] H.K. Xu, A regularization method for the proximal point algorithms, J. Global Optim., 36 (2006), 115-125. doi: 10.1007/s10898-006-9002-7. 